\title{
2001113818
}

J. Quant. Spectrosc. Radiat. Transfer, in press (2001)

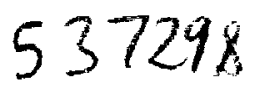

$10 f_{5}$

\section{Radiation force caused by scattering, absorption, and emission of light by nonspherical particles}

\author{
Michael I. Mishchenko*
}

NASA Goddard Institute for Space Studies, 2880 Broadway, New York, NY 10025, USA

\begin{abstract}
General formulas for computing the radiation force exerted on arbitrarily oriented and arbitrarily shaped nonspherical particles due to scattering, absorption, and emission of electromagnetic radiation are derived. For randomly oriented particles with a plane of symmetry, the formula for the average radiation force caused by the particle response to external illumination reduces to the standard Debye formula derived from the Lorenz-Mie theory, whereas the average radiation force caused by emission vanishes.
\end{abstract}

Keywords: Radiation force, Electromagnetic scattering and emission; Nonspherical particles

*Tel.: +1-212-678-5590; fax: +1-212-678-5622.

E-mail address: crmim@giss.nasa.gov (M. I. Mishchenko). 
It is well known that a particle illuminated by a beam of light experiences a force called radiation pressure. This phenomenon is used in laboratories to levitate and size small particles $[1,2]$ and affects the spatial distribution of interstellar and circumstellar dust grains [3-5]. In a renowned paper published in 1909 [6], Debye showed that the radiation force exerted on a spherical particle is directed along the propagation direction of the incident beam and derived a simple formula expressing the magnitude of the force in terms of the particle extinction and scattering cross sections and the asymmetry parameter. This Note extends Debye's analysis to arbitrarily oriented and arbitrarily shaped nonspherical particles. In addition, it considers the radiation force caused by emission of electromagnetic radiation by a nonspherical particle having a finite absolute temperature.

Let an arbitrary particle be illuminated by a time-harmonic parallel electromagnetic wave. If the amplitudes of the incident and scattered fields do not change in time, the force due to radiation pressure exerted on the particle averaged over the period $2 \pi / \omega$ of the incident wave is

$$
\mathbf{F}_{1}=\int_{S} \mathrm{~d} S\left\langle\vec{T}_{\mathrm{M}}(\mathbf{r})\right\rangle \cdot \hat{n}
$$

(Section 6.7 of [7]), where $\mathbf{r}$ is the position vector, $\bar{T}_{\mathrm{M}}$ is the Maxwell stress tensor, the integration is performed over a closed surface $S$ surrounding the scattering object, and $\hat{n}$ is the unit vector in the direction of the local outward normal to $S$. Assume for simplicity that the scattering object is imbedded in a vacuum. Then the instantaneous value of the Maxwell stress tensor is [7]

$$
\begin{aligned}
\bar{T}_{\mathrm{M}}(\mathbf{r}, t) & =\varepsilon_{0}\left[\mathbf{E}(\mathbf{r}, t) \otimes \mathbf{E}(\mathbf{r}, t)+c^{2} \mathbf{B}(\mathbf{r}, t) \otimes \mathbf{B}(\mathbf{r}, t)-\frac{1}{2}\left[\mathbf{E}(\mathbf{r}, t) \cdot \mathbf{E}(\mathbf{r}, t)+c^{2} \mathbf{B}(\mathbf{r}, t) \cdot \mathbf{B}(\mathbf{r}, t)\right] \bar{I}\right] \\
& =\varepsilon_{0} \mathbf{E}(\mathbf{r}, t) \otimes \mathbf{E}(\mathbf{r}, t)+\mu_{0} \mathbf{H}(\mathbf{r}, t) \otimes \mathbf{H}(\mathbf{r}, t)-\frac{1}{2}\left[\varepsilon_{0} \mathbf{E}(\mathbf{r}, t) \cdot \mathbf{E}(\mathbf{r}, t)+\mu_{0} \mathbf{H}(\mathbf{r}, t) \cdot \mathbf{H}(\mathbf{r}, t)\right] \bar{I},
\end{aligned}
$$

where $\mathbf{E}$ is the electric and $\mathbf{H}$ the magnetic field, $\mathbf{B}$ is the magnetic induction, $\varepsilon_{0}$ is the electric permittivity and $\mu_{0}$ the magnetic permeability of free space, $c=1 / \sqrt{\varepsilon_{0} \mu_{0}}$ is the speed of light in 
a vacuum, $\bar{I}$ is the identity dyad, and $\otimes$ denotes a dydic product of two vectors. The time average of the Maxwell stress tensor is

$$
\left\langle\vec{T}_{\mathbf{M}}(\mathbf{r})\right\rangle=\frac{1}{2} \operatorname{Re}\left[\varepsilon_{0} \mathbf{E}(\mathbf{r}) \otimes \mathbf{E}^{*}(\mathbf{r})+\mu_{0} \mathbf{H}(\mathbf{r}) \otimes \mathbf{H}^{*}(\mathbf{r})-\frac{1}{2}\left(\varepsilon_{0}|\mathbf{E}(\mathbf{r})|^{2}+\mu_{0}|\mathbf{H}(\mathbf{r})|^{2}\right) \bar{I}\right],
$$

where * denotes a complex-conjugate value. It is convenient to choose for $S$ a sphere centered at the scattering object and having a radius $r$ big enough to be in the far-field zone so that the scattered wave is spherical [7]. Then Eq. (1) becomes

$$
\mathbf{F}_{1}=r^{2} \int_{4 \pi} \mathrm{d} \hat{r}\left\langle\vec{T}_{\mathrm{M}}(r \hat{r})\right\rangle \cdot \hat{r}
$$

where $\hat{r}=\mathbf{r} / r$.

The total electric and magnetic fields at $\mathbf{r}$ are vector sums of the respective incident ("inc") and scattered ("sca") components given by [8]

$$
\begin{aligned}
& \mathbf{E}^{\text {inc }}(\mathbf{r})=\mathbf{E}_{0}^{\text {inc }} \exp \left(i k \hat{n}^{\text {inc }} \cdot \mathbf{r}\right) \\
& \underset{r \rightarrow \infty}{=} \frac{2 \pi i}{k}\left[\delta\left(\hat{n}^{\mathrm{inc}}+\hat{r}\right) \frac{e^{-i k r}}{r}-\delta\left(\hat{n}^{\mathrm{inc}}-\hat{r}\right) \frac{e^{i k r}}{r}\right] \mathbf{E}_{0}^{\mathrm{inc}}, \quad \mathbf{E}_{0}^{\mathrm{inc}} \cdot \hat{n}^{\mathrm{inc}}=0, \\
& \mathbf{H}^{\mathrm{inc}}(\mathbf{r})=\frac{1}{i \omega \mu_{0}} \nabla \times \mathbf{E}^{\mathrm{inc}}(\mathbf{r}) \\
& =\sqrt{\frac{\varepsilon_{0}}{\mu_{0}}} \exp \left(i k \hat{n}^{\mathrm{inc}} \cdot \mathbf{r}\right) \hat{n}^{\mathrm{inc}} \times \mathbf{E}_{0}^{\mathrm{inc}} \\
& \underset{r \rightarrow \infty}{=} \frac{2 \pi i}{k}\left[\delta\left(\hat{n}^{\mathrm{inc}}+\hat{r}\right) \frac{e^{-i k r}}{r}-\delta\left(\hat{n}^{\mathrm{inc}}-\hat{r}\right) \frac{e^{i k r}}{r}\right] \sqrt{\frac{\varepsilon_{0}}{\mu_{0}}} \hat{n}^{\mathrm{inc}} \times \mathbf{E}_{0}^{\mathrm{inc}}, \\
& \mathbf{E}^{\mathrm{sca}}(\mathbf{r}) \underset{r \rightarrow \infty}{=} \frac{e^{i k r}}{r} \mathbf{E}_{\mathrm{l}}^{\mathrm{sca}}(\hat{r}), \quad \mathbf{E}_{\mathrm{l}}^{\mathrm{sca}}(\hat{r}) \cdot \hat{r}=0, \\
& \mathbf{H}^{\mathrm{sca}}(\mathbf{r})=\frac{1}{i \omega \mu_{0}} \nabla \times \mathbf{E}^{\mathrm{sca}}(\mathbf{r}) \\
& \underset{r \rightarrow \infty}{=} \sqrt{\frac{\varepsilon_{0}}{\mu_{0}}} \frac{e^{i k r}}{r} \hat{r} \times \mathrm{E}_{1}^{\mathrm{sca}}(\hat{r})
\end{aligned}
$$

where $i=\sqrt{-1}, k=\omega \sqrt{\varepsilon_{0} \mu_{0}}$ is the wave number in the surrounding medium, and $\hat{n}^{\text {inc }}$ is the 
unit vector in the incidence direction. Because the incident and scattered fields are transverse, the first and second terms in square brackets on the right-hand side of Eq. (3) do not contribute to the integral in Eq. (4). We thus have

$$
\begin{aligned}
\mathbf{F}_{1}= & -\frac{\varepsilon_{0} r^{2}}{4} \operatorname{Re} \int_{4 \pi} \mathrm{d} \hat{r} \hat{r}\left\{\left|\mathbf{E}^{\mathrm{inc}}(\mathbf{r})\right|^{2}+\left|\mathbf{E}^{\mathrm{sca}}(\mathbf{r})\right|^{2}+2 \mathbf{E}^{\mathrm{inc}}(\mathbf{r}) \cdot\left[\mathbf{E}^{\mathrm{sca}}(\mathbf{r})\right]^{*}\right\} \\
& -\frac{\mu_{0} r^{2}}{4} \operatorname{Re} \int_{4 \pi} \mathrm{d} \hat{r} \hat{r}\left\{\left|\mathbf{H}^{\mathrm{inc}}(\mathbf{r})\right|^{2}+\left|\mathbf{H}^{\mathrm{sca}}(\mathbf{r})\right|^{2}+2 \mathbf{H}^{\mathrm{inc}}(\mathbf{r}) \cdot\left[\mathbf{H}^{\mathrm{sca}}(\mathbf{r})\right]^{*}\right\} .
\end{aligned}
$$

The terms $\left|E^{\text {inc }}(\mathbf{r})\right|^{2}$ and $\left|\mathbf{H}^{\text {inc }}(\mathbf{r})\right|^{2}$ are constants independent of $\mathbf{r}$, and their contribution to $\mathbf{F}_{1}$ is simply zero. The contribution of the remaining terms follows from Eqs. (5)-(8) and the vector identity $(\mathbf{a} \times \mathbf{b}) \cdot(\mathbf{c} \times \mathbf{d})=(\mathbf{a} \cdot \mathbf{c})(\mathbf{b} \cdot \mathbf{d})-(\mathbf{a} \cdot \mathbf{d})(\mathbf{b} \cdot \mathbf{c})$ :

$$
\mathbf{F}_{1}=\frac{2 \pi \varepsilon_{0}}{k} \hat{n}^{\mathrm{inc}} \operatorname{Im}\left[\mathbf{E}_{1}^{\mathrm{sca}}\left(\hat{n}^{\mathrm{inc}}\right) \cdot \mathbf{E}_{0}^{\mathrm{inc} *}\right]-\frac{\varepsilon_{0}}{2} \int_{4 \pi} \mathrm{d} \hat{r} \hat{r}\left|\mathbf{E}_{1}^{\mathrm{sca}}(\hat{r})\right|^{2}
$$

Recalling the extinction theorem [8] and the definitions of the extinction cross section $C_{\text {ext }}$, differential scattering cross section $\frac{\mathrm{d} C_{\mathrm{sca}}}{\mathrm{d} \Omega}$ [9], and phase matrix $\mathbf{Z}$ [10], we finally derive

$$
\begin{aligned}
& \mathbf{F}_{1}=\frac{1}{c} \hat{n}^{\text {inc }} C_{\text {ext }} I^{\text {inc }}-\frac{I^{\text {inc }}}{c} \int_{4 \pi} \mathrm{d} \hat{r} \hat{r} \frac{\mathrm{d} C_{\text {sca }}}{\mathrm{d} \Omega} \\
&=\frac{1}{c} \hat{n}^{\text {inc }} C_{\text {ext }} I^{\text {inc }}-\frac{1}{c} \int_{4 \pi} \mathrm{d} \hat{r} \hat{r}[ Z_{11}\left(\hat{r}, \hat{n}^{\text {inc }}\right) I^{\text {inc }}+Z_{12}\left(\hat{r}, \hat{n}^{\text {inc }}\right) Q^{\text {inc }} \\
&\left.+Z_{13}\left(\hat{r}, \hat{n}^{\text {inc }}\right) U^{\text {inc }}+Z_{14}\left(\hat{r}, \hat{n}^{\text {inc }}\right) V^{\text {inc }}\right],
\end{aligned}
$$

where $I^{\text {inc }}=\frac{1}{2} \sqrt{\frac{\varepsilon_{0}}{\mu_{0}}}\left|\mathbf{E}_{0}\right|^{2}$ is the intensity of the incident beam.

Although the first term on the right-hand side of $\mathrm{Eq}$. (11) represents a force component in the direction of $\hat{n}^{\text {inc }}$, the direction of the total force is, in general, different from the direction of propagation of the incident beam and depends on its polarization state because of the second term. The projection of the total force on any direction $\hat{n}$ is simply the dot product $\mathbf{F}_{1} \cdot \hat{n}$. In particular, the component of the force in the incidence direction is 


$$
\begin{aligned}
\mathrm{F}_{1} \cdot \hat{n}^{\mathrm{inc}} & =\frac{1}{c} C_{\mathrm{ext}} I^{\mathrm{inc}}-\frac{I^{\mathrm{inc}}}{c} \int_{4 \pi} \mathrm{d} \hat{r} \hat{r} \cdot \hat{n}^{\mathrm{inc}} \frac{\mathrm{d} C_{\text {sca }}}{\mathrm{d} \Omega} \\
& =\frac{1}{c} I^{\mathrm{inc}}\left[C_{\mathrm{ext}}-C_{\mathrm{sca}}\langle\cos \Theta\rangle\right] \\
& =\frac{1}{c} I^{\mathrm{inc}} C_{\mathrm{pr}},
\end{aligned}
$$

where

$$
\langle\cos \Theta\rangle=\frac{1}{C_{\text {sca }}} \int_{4 \pi} \mathrm{d} \hat{r} \frac{\mathrm{d} C_{\text {sca }}}{\mathrm{d} \Omega} \hat{r} \cdot \hat{n}^{\text {inc }}
$$

is the asymmetry parameter,

$$
C_{\mathrm{sca}}=\int_{4 \pi} \mathrm{d} \hat{r} \frac{\mathrm{d} C_{\mathrm{sca}}}{\mathrm{d} \Omega}
$$

is the total scattering cross section, and the quantity

$$
C_{\mathrm{pr}}=C_{\mathrm{ext}}-C_{\mathrm{sca}}\langle\cos \Theta\rangle
$$

is the cross section for radiation pressure.

Although being the result of a lengthy rigorous derivation, Eq. (12) allows a transparent physical interpretation. A beam of light carries linear momentum as well as energy. The direction of the momentum is that of propagation, while the absolute value of the momentum is energy/(speed of light). Since the total momentum of the electromagnetic field and the scattering object must be constant, the radiation force exerted on the object is equal to the momentum removed from the total electromagnetic field per unit time. Consider the component of the force in the direction of incidence. The momentum removed from the incident beam per unit time is $C_{\text {ext }} I^{\text {inc }} / c$. Of this amount, the part due to absorption is not replaced, whereas the part due to scattering is partially replaced by the contribution due to the projection of the moment of the scattered light on the direction of incidence. This contribution is equal to the integral of $I^{\text {sca }} \cos \Theta / c$ over all scattering directions, or $I^{\text {inc }} C_{\text {sca }}\langle\cos \Theta\rangle / c$. Note that van de Hulst [11] used these arguments as a heuristic derivation of Eq. (12).

If the absolute temperature of the particle $T$ is above zero, then electromagnetic radiation 
emitted by the particle causes an additional component of the radiation force. The emitted radiation is incoherent and does not interact with the incident and scattered radiation, thereby generating an independent radiation force component. Furthermore, emission is analogous to scattering in that it generates radiation propagating radially in all directions. Therefore, we can write the emission component of the radiation force by analogy with the scattering component given by the second term on the right-hand side of Eq. (11):

$$
\mathbf{F}_{2}(T)=-\frac{1}{c} \int_{0}^{\infty} \mathrm{d} \omega \int_{4 \pi} \mathrm{d} \hat{r} \hat{r} \mathrm{~K}_{\mathrm{el}}(\hat{r}, T, \omega)
$$

where $\mathrm{K}_{\mathrm{el}}(\hat{r}, T, \omega)$ is the first element of the Stokes emission vector. $\mathrm{K}_{\mathrm{e} 1}(\hat{r}, T, \omega)$ is the amount of electromagnetic energy emitted by the particle in the direction $\hat{r}$ per unit solid angle per unit frequency interval per unit time and is expressed in terms of the particle extinction, $\mathbf{K}$, and phase, $\mathbf{Z}$, matrices as follows [10]:

$$
\mathrm{K}_{\mathrm{el}}(\hat{r}, T, \omega)=I_{\mathrm{b}}(T, \omega) \mathrm{K}_{11}(\hat{r}, \omega)-I_{\mathrm{b}}(T, \omega) \int_{4 \pi} \mathrm{d} \hat{r}^{\prime} Z_{11}\left(\hat{r}, \hat{r}^{\prime}, \omega\right)
$$

where

$$
I_{\mathrm{b}}(T, \omega)=\frac{1}{4} \pi^{-3} c^{-2} \hbar \omega^{3}\left[\exp \left(\frac{\hbar \omega}{k_{\mathrm{B}} T}\right)-1\right]^{-1}
$$

is the Planck blackbody energy distribution [9]. [Note that the particle is assumed to be isothermal, which should be appropriate for micron-sized particles exposed to radiation sources of moderate strength.] Unlike $\mathbf{F}_{1}$, the emission component of the radiation force depends on the particle temperature.

The total radiation force exerted on the particle is the vector sum of the component caused by the particle response to the external illumination and the component caused by emission: $\mathbf{F}=\mathbf{F}_{1}+\mathbf{F}_{2}$. This force depends on the direction and polarization state of the incident light and on the particle orientation, shape, size, refractive index, and temperature. The optical characteristics of the particle entering Eqs. (11) and (16) can be computed using one of the existing theoretical techniques based on solving the macroscopic Maxwell equations or their 
integral counterparts [10].

Note that for particles in a vacuum, the particle temperature is linked to the incident radiation because the total absorption of electromagnetic radiation by a particle must be balanced by the total particle emission. In this sense, the forces $F_{1}$ and $F_{2}$ are coupled - both arise from the incident radiation.

Consider now a small volume element comprising randomly oriented particles with a plane of symmetry (such as spheres or spheroids) or particles and their mirror counterparts in equal numbers and in random orientation. Following the analysis of symmetries performed by van de Hulst [11], it is straightforward to show that the emission component of the radiation force vanishes, whereas the component caused by the external illumination is now directed along $\hat{n}^{\text {inc }}$, is independent of the polarization state of the incident beam, and is given by

$$
\begin{aligned}
\left\langle\mathbf{F}_{1}\right\rangle & =\frac{1}{c} \hat{n}^{\mathrm{inc}} I^{\mathrm{inc}}\left[\left\langle C_{\mathrm{ext}}\right\rangle-\left\langle C_{\mathrm{sca}}\right\rangle\langle\cos \Theta\rangle\right] \\
& =\frac{1}{c} \hat{n}^{\mathrm{inc}} I^{\mathrm{inc}}\left\langle C_{\mathrm{pr}}\right\rangle,
\end{aligned}
$$

where $\left\langle C_{\text {ext }}\right\rangle,\left\langle C_{\text {sca }}\right\rangle$, and $\left\langle C_{\mathrm{pr}}\right\rangle$ are the orientation-averaged extinction, scattering, and radiation-pressure cross sections, respectively. This formula is identical to that derived by Debye [6] for homogeneous spherical particles.

Finally we note that the radiation pressure caused by external illumination is accompanied by the radiation torque exerted on the particle and given by

$$
\begin{aligned}
\Gamma_{1} & =-\int_{S} \mathrm{~d} S r \hat{r} \cdot\left[\left\langle\vec{T}_{\mathrm{M}}(\mathbf{r})\right\rangle \times \hat{r}\right] \\
& =-r^{3} \int_{4 \pi} \mathrm{d} \hat{r} \hat{r} \cdot\left[\left\langle\bar{T}_{\mathrm{M}}(\mathbf{r})\right\rangle \times \hat{r}\right]
\end{aligned}
$$

(cf. page 288 of [7]), where $r$ is the radius of a sphere $S$ centered inside the scattering particle and having its surface in the far-field zone. Since $\hat{r} \cdot \bar{I} \times \hat{r}$ vanishes identically, only the first two terms in square brackets on the right-hand side of Eq. (3) contribute to the integrals in Eq. (19). The evaluation of this contribution is complicated because it requires the knowledge of not only 
the transverse component of the scattered electric and magnetic fields, but also of the longitudinal component, which we have so far neglected because it decays faster than $1 / r$. Marston and Crichton [12] computed $\Gamma_{1}$ for homogeneous and isotropic spherical particles, whereas Draine and Weingartner [3] derived a formula for $\Gamma_{1}$ in the framework of the discrete dipole approximation. Emission generates an additional component of the radiation torque independent of $\Gamma_{1}$.

\section{Acknowledgments}

I appreciate very instructive reviewer's comments on a preliminary version of this paper.

This research was sponsored by the NASA Radiation Science Program managed by Donald Anderson.

\section{References}

[1] Ashkin A, Dziedzic J. Observation of light scattering from nonspherical particles using optical levitation. Appl Opt 1980; 19: 660-668.

[2] Chýlek P, Ngo D, Pinnick RG. Resonance structure of composite and slightly absorbing spheres. J Opt Soc Am A 1992; 9: 775-780.

[3] Draine BT, Weingartner JC. Radiative torques on interstellar grains. I. Superthermal spin-up. Astrophys J 1996; 470: 551-565.

[4] Il'in VB, Voshchinnikov NN. Radiation pressure on non-spherical dust grains in envelopes of late-type giants. Astron. Astrophys. Suppl. 1998; 128: 187-196.

[5] Landgraf M, Augustsson K, Grun E, Gustafson BÅS. Deflection of the local interstellar dust flow by solar radiation pressure. Science 1999; 286: 2319-2322.

[6] Debye P. Der Lichtdruk auf Kugeln von beliebigem Material. Ann. Physik 1909; 30: 57-136.

[7] Jackson JD. Classical electrodynamics. New York: Wiley, 1998.

[8] Saxon DS. Lectures on the scattering of light. Los Angeles: Univ. of California, 1955. 
[9] Bohren CF, Huffman DR. Absorption and scattering of light by small particles. New York, Wiley, 1983.

[10] Mishchenko MI, Hovenier JW, Travis LD, Eds. Light scattering by nonspherical particles: theory, measurements, and applications. San Diego: Academic Press, 2000.

[11] van de Hulst HC. Light scattering by small particles. New York, Wiley, 1957.

[12] Marston PL, Crichton JH. Radiation torque on a sphere caused by a circularly-polarized electromagnetic wave. Phys. Rev. A 1984; 30: 2508-2516. 
